type=other in-section=minerva id=minpic090115 in-journal=bmj elocation-id=h6661

doi=10.1136/bmj.h6661

\title{
Unusual presentation of a contained AAA rupture
}

Shavini Weerasekera[a1]

foundation year 1 doctor

Sanja Thompson[a1]

consultant geriatrician

Raman Uberoi[a2]

consultant radiologist

A1 Department of Geratology, John Radcliffe Hospital, Oxford, UK

A2 Department of Radiology, John Radcliffe Hospital

Correspondence to: S Thompson sanja.thompson@ouh.nhs.uk

Figure - minpic090115.f1

A 90 year old man was transferred to the rehabilitation ward with poor mobility, lower body oedema, and raised inflammatory markers. He initially presented with abdominal pain and haematemesis attributed to severe gastro-oesophagitis. Contrast enhanced computed tomography showed a contained rupture of a $6.6 \mathrm{~cm}$ saccular infrarenal abdominal aneurysm with large heterogeneous para-aortic haematoma. A cystic area showed rim enhancement[f1] consistent with infection. Surgery was not appropriate because of frailty. However, he improved with antibiotics and was discharged. About $4 \%$ of ruptured abdominal aortic aneurysms are contained ("sealed") and can be missed. Presentation may include increased inflammatory markers, from superimposed haematomal infection, and severe lower body oedema, probably because of inferior vena cava compression and lymphatic obstruction. Patient consent obtained.

Cite this as: $B M J$ 2015;351:h6661 